EGU2020-7168

https://doi.org/10.5194/egusphere-egu2020-7168

EGU General Assembly 2020

(c) Author(s) 2020. This work is distributed under

the Creative Commons Attribution 4.0 License.

\title{
Sentinel products assimilation in a complete hydro/fire- meteorological chain: nearly operational experiments in the framework of the E-SHAPE project
}

\author{
Martina Lagasio ${ }^{1}$, Agostino N. Meroni ${ }^{1}$, Luca Pulvirenti ${ }^{1}$, Giuseppe Squicciarino ${ }^{1}$, Antonio Parodi ${ }^{1}$, \\ Alexia Tsouni ${ }^{2}$, Haris Kontoes ${ }^{2}$, and Nikos Bartsotas ${ }^{2}$ \\ ${ }^{1}$ CIMA Research Foundation, Savona, Italy (martina.lagasio@cimafoundation.org) \\ ${ }^{2}$ National Observatory of Athens, Greece
}

In the framework of the E-SHAPE "EuroGEO Showcase: Applications Powered by Europe" project, the Pilot 2 application of the Disasters Resilience Showcase concerns the disasters in urban environment. Starting from the results and methodologies analyzed in the framework of the STEAM project, the E-SHAPE pilot exploits the new capacities for designing and delivering innovative services for extreme-scale fire/hydro-meteorological modelling chain assimilating Copernicus data and core services directly ingested through the Copernicus Open Access Hub APIS, and the DIAS platform, as well as citizen scientists data, to enable more precise predictions and decision-making support for high impact events in urban and peri urban environment. Contributing to the Disaster Resilience SBA, one of the main activities listed in the GEO Space and Security Community Activity is to get maximum benefit from the use of large and heterogeneous datasets to potentially fill in the observational and capability gaps at EU decision making level. To this end, the application proposes also the integration of the datasets and tools made available in the frame of the pilot application (weather, citizen science, hydrological and fire models included in CIMA's Platforms Dewetra and RASOR and NOA's BEYOND Systems FireHub and FloodHub) for the impact assessment of natural hazards over areas of interest with regard to human security issues. An example of innovative service is the ingestion of high-resolution Copernicus remote sensing products in Numerical Weather Prediction (NWP) models. The rationale is that NWP models are presently able to produce forecasts with a spatial resolution in the order of $1 \mathrm{~km}$, but unreliable surface information or poor knowledge of the initial state of the atmosphere may imply an inaccurate simulation of the weather phenomena. It is expected that forecast inaccuracies could be reduced by ingesting high resolution Earth Observation products into the models. In this context, the Copernicus Sentinel satellites represent an important source of data, because they can provide a set of high-resolution observations of physical variables (e.g. soil moisture, land/sea surface temperature, wind over sea, columnar water vapor) used in NWP model runs. The possible availability of a spatially dense Personal Weather Stations network could also be exploited to allow NWP models to assimilate timely updated data such as temperature, humidity and pressure. In this work a preliminary experiment design and methodology will be presented. 
How to cite: Lagasio, M., Meroni, A. N., Pulvirenti, L., Squicciarino, G., Parodi, A., Tsouni, A., Kontoes, $\mathrm{H}_{\text {., }}$ and Bartsotas, N.: Sentinel products assimilation in a complete hydro/firemeteorological chain: nearly operational experiments in the framework of the E-SHAPE project, EGU General Assembly 2020, Online, 4-8 May 2020, EGU2020-7168,

https://doi.org/10.5194/egusphere-egu2020-7168, 2020 